\title{
E-LEARNING ISSUES FOR ADVANCED TECHNICAL TOPICS
}

The case of Electronic Design Automation training

Fulvio Corno

Politecnico di Torino, Italy

Abstract: $\quad$ This paper describes the problems found in applying e-learning methodologies and tools to an advanced technical topic: training in Electronic Design Automation at the University level. Several problems are identified, that lie in the critical path to successful implementation of learning programs for future electrical and computer engineers.

Key words: Electronic Design Automation; E-learning.

\section{INTRODUCTION}

The social and economical impacts of e-learning suggest its application to several learning ages and knowledge fields. In this section, we will concentrate on teaching technical topics to higher-education students.

At Politecnico di Torino, several degrees in Engineering are being taught with distance learning technologies (computer engineering, electronic eng., telecommunications eng., logistics and management eng., mechanical eng. and electrical eng.), using three different methodologies:

- Completely on-line courses, where students receive lectures recorded on VHS tapes or Video CDs, and find additional study material and practical assignment texts and solutions on a web site. Students are followed by a tutor, who is available over the phone or by e-mail, and can participate in a mailing list with other students enrolled in the same course. The tutor also organizes in-presence sessions, where students may travel to the University and review some theoretical concepts, solve some exercises 
proposed by the tutor, or ask for clarifications on any issues. You may find further information at the URL http://corsiadistanza.polito.it.

- Blended approach, that we apply on a remote site located in a rural area of Sardinia: students have great incentives to get a technical degree in order to find work, but bad logistics and transportation costs prevent many of them from attending university. Therefore, Politecnico di Torino opened a Learning Center (Centro Multimediale Montiferro) where students must be physically present every afternoon, and they are assisted by local tutors (not university professors, but technical workers, highschool teachers, etc). The learning sessions may be of 4 types: (a) videoconference with a University teacher from Politecnico di Torino, for dealing with some theoretical topic, (b) vision of recorded video lectures, re-using the same material available for completely on-line courses, but with the assistance of the local tutor that may answer questions in real time or clarify specific points, (c) solving exercises and assignments with the help of the local tutors, (d) videoconference with a University teacher from Politecnico di Torino, for a question-and-answer session. You may find further information at the URL http://www.montiferru.it.

- Supporting traditional courses through on-line material. In this case, traditional university courses are supplemented my additional study material, lecture notes, exercises, past exams, etc, available over the Internet. There is a strong effort from the Faculties to keep the study program as much aligned as possible (compatibly with the freedom of individual teachers) in order to be able to reuse materials produced for on-line courses as support material for traditional courses, and vice-versa. You may find further information at the URL http://didattica.polito.it.

Experience over several years suggest that the potentials for e-learning are great, but we must face several difficulties to make it effective. In the following, we will concentrate mainly on problems arising in advanced technical topics. As a case study, we will analyze training in electronic design automation (EDA) field.

\section{CASE STUDY: CHARACTERISTICS OF EDA TRAINING}

EDA is a very important topic for engineers in ICT (computer engineers, electrical engineers), as it is the basis for the design of all current electronic devices. The complexity of current electronic circuits, ranging in the orders of tens or hundreds of millions of transistors, completely prevents manual design: circuit designers must use advanced automatic tools to help them 
design, simulate, implement their chips. Such tools are constantly lagging behind the complexity of circuits, so using the tools is a challenge by itself, and involves combining design knowledge and tool experience.

These requirements imply that Electronic Design Automation students must become proficient in three main areas:

- Understanding and designing complex algorithms and data structures, coping with information size (e.g., graph structures with millions of nodes) and with the intractability of the problems (i.e., NP-complete algorithms for almost any problem of practical interest). Students are required to gain competence about algorithm design and complexity, the most used complex data structures, and to develop intuition about the trade-off between algorithm complexity (running time and required memory) and the quality of the result (circuit optimization).

- Understanding the design process of VLSI circuits. This is a complex flow, made of many steps, describing the circuit starting from a high level representation and progressively adding lower level detail, and ranging through: hardware-software partitioning, architectural design, interface and protocol definition, scheduling and allocation, registertransfer level design, logic synthesis, timing optimization, circuit layout, area optimization. Each step is followed by a verification or validation step, usually through simulation of the design. At each step the designer uses different tools, describes the circuit using different languages (graphical or textual), and simulates with different models. Student should gain experience about the problems of each phase, know what tools and languages are used, and understand the limitations of tools.

- Designing a circuit. This means being able to carry out the several phases of the design process, and understanding the design methodology at each of the description levels. Usually, students specialize on some level (high-level versus low-level areas of the design flow), but curricula tend to expose all students to all levels, at least on some small-scale circuit.

Teaching methodologies involve mixing three levels: theoretical information (algorithms, design flow, design methods), practical exercises (algorithm implementation, circuit designs), and usage of the tools in a laboratory setting.

\section{E-LEARNING FOR EDA}

How can this experience be transferred to e-learning? Can state-of-the art teaching methodologies and LMS implementations support the students in this hard, complex, yet essential discipline? 
Current e-learning solutions appear insufficient for this knowledge domain. Generally speaking, the "theoretical" and the "methodological" parts of EDA can be easily transferred to an on-line learning solution. However, the practical parts, involving design exercises and tool usage, still pose incredible challenges. Some of the major issues are raised below.

Concerning algorithm design and implementation, how can we help the students developing their intuition and capability to evaluate trade-offs? In the context of EDA, programming skills are not enough, since due to the complexity of the problems much relies on experience, intuition, trial-anderror and experimentation, hardly supported by existing e-learning solutions.

On the other hand, developing design experience usually requires both acquiring methodological information, which can be easily included in LMS contents, and practical skill deriving from trying to design several circuits or circuit parts, of increasing complexity. The LMS may help in providing the necessary infrastructure for storing the design exercised and for delivering them in the right complexity progression, by supporting the personalized learning curve of the student. However, current automatic evaluation solutions are not enough. No single solution exists for a design exercise: each student may develop a different, yet completely correct, design solution. Manual correction of such exercises is still necessary, and this involves heavy burdens on the facilitators and tutors.

Finally, the third challenge is on gaining experience andfamiliarity with the tools. This involves letting the students to use a set of tools that should be comparable to the ones currently used in the EDA industry. There are three main problems to overcome. First, the cost of the tools: such software is priced towards the industry needs and financial capabilities. Universities can get limited educational versions, for use in university laboratories, but licenses usually prevent remote usage by on-line students. Second, minimum hardware requirements by EDA tools (operating system, available memory, and processor type) are usually much higher that common PCs owned by students. Licensing problems aside, it would be impossible to run these tools on students' machines. Third, tools are not integrated with the learning management system: the learner should switch between the LMS and the EDA tool while learning to use the tool, and later he or she would need to use the EDA tool to solve some exercises proposed by the LMS.

The whole situation is made more complex by the rapid evolution of involved technologies: both VLSI technology (and the associated design flows) and EDA technology (and the associated design tools) evolve rapidly, with new versions or new innovations every semester. This makes it difficult and more expensive to capitalize the production of learning material or the deployment of virtual laboratory settings. 
On the positive side, there are several experiences of successful use of elearning in limited EDA contexts. Most EDA tool vendors offer e-learning solutions to learn their tools: such courses replace or supplement training seminars needed when new tools are introduced in industry practice. Such solutions are cost-effective and appreciated in industrial contexts (on learning-on-the-job settings), since individual designers may study on their spare time and need not synchronize their agendas. Furthermore, most learners already have design experience or used similar tools in the past. The same courses are not directly suitable in university contexts, due both to the limited design knowledge of the students and the necessity of learning several tools from different vendors, whose learning management systems are not integrated.

Some universities have also taken the challenge, and are offering EDA courses on-line. Some are offering custom solution to be able to use EDA tools (either thanks to limited educational PC versions or through remote connectivity with university servers). Some rely on the collaboration capabilities of the LMS to help the student develop their designs in a joint fashion, under the supervision of a facilitator. In fact, it appears that the only viable solutions are the adoption of advanced collaboration frameworks, allowing application sharing, and collaborative problem solving, sketching capabilities, and working primarily in the synchronous mode.

\section{CONCLUSIONS}

The adoption of e-learning models, supported by effective learning management systems, is crucial to cover the demand for skilled engineers in circuits and systems design. However, the vastness of this knowledge domain, the requirement for strong practical experiences, and the complexity of the involved software tools are posing great challenges for the successful implementation of study programs. Universities, industry learning centers, and EDA tool vendors are all struggling with the development of learning models, and the associated learning tools, that may help engineering students and employed designers to gain sufficient skills in this crucial area for ICT development. Effective solutions for e-learning in advanced technical topics are still open problems. Instructors and LMS developers should join and try to solve the fundamental issues of teaching intuition, design mentality, and sophisticated tool usage. 


\section{REFERENCES}

1. C.Anderson, M. Brennan, eLearning in Practice - Proprietary Knowledge and Instructional Design, IDC, 2001, http://www.e-learningsite.com/download/white/isd-idc.pdf

2. D. Jansen, The Electronic Design Automation Handbook, Kluwer Academic Publishers, 2003 